\title{
Clustered Wigner-crystal phases of cold polar molecules in arrays of one-dimensional tubes
}

\section{Citation}

Knap, Michael, Erez Berg, Martin Ganahl, and Eugene Demler. 2012. “Clustered Wigner-Crystal Phases of Cold Polar Molecules in Arrays of One-Dimensional Tubes." Physical Review B 86 (6). https://doi.org/10.1103/physrevb.86.064501.

\section{Permanent link}

http://nrs.harvard.edu/urn-3:HUL.InstRepos:41412225

\section{Terms of Use}

This article was downloaded from Harvard University's DASH repository, and is made available under the terms and conditions applicable to Open Access Policy Articles, as set forth at http:// nrs.harvard.edu/urn-3:HUL.InstRepos:dash.current.terms-of-use\#OAP

\section{Share Your Story}

The Harvard community has made this article openly available.

Please share how this access benefits you. Submit a story.

Accessibility 


\title{
Clustered Wigner crystal phases of cold polar molecules in arrays of one-dimensional tubes
}

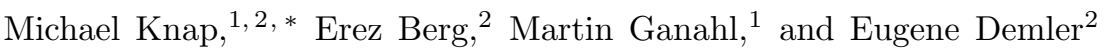 \\ ${ }^{1}$ Institute of Theoretical and Computational Physics, \\ Graz University of Technology, 8010 Graz, Austria \\ ${ }^{2}$ Department of Physics, Harvard University, Cambridge MA 02138, USA
}

(Dated: September 18, 2018)

\begin{abstract}
We analyze theoretically polar molecules confined in planar arrays of one dimensional tubes. In the classical limit, if the number of tubes is finite, new types of "clustered Wigner crystals" with increasingly many molecules per unit cell can be stabilized by tuning the in-plane angle between the dipolar moments and the tube direction. Quantum mechanically, these phases melt into distinct "clustered Luttinger liquids." We calculate the phase diagram of the system and study the quantum melting of the clustered phases. We find that the requirements for exploring these phases are reachable in current experiments and discuss possible experimental signatures.
\end{abstract}

PACS numbers: 05.30.-d, 03.75.Hh, 67.85.-d, 34.20.-b

\section{INTRODUCTION}

Systems with competing long-range interactions often exhibit structures with emergent large length scales. Some examples include the formation of bubble and stripe domains in Langmuir-Blodgett films or in thin ferromagnetic layers, $\frac{112}{112}$ and the chain formation of magnetic particles in three-dimensional ferrofluids. ${ }^{3}$ Long-range dipolar interactions in a back-gated two-dimensional electron gas (2DEG) have been predicted ${ }^{45}$ to lead to the existence of "microemulsion" phases intervening the Fermi liquid and the Wigner crystal phase. Similar microemulsion phases may appear in 2DEGs subject to magnetic fields such that several Landau levels are occupied 6 [6

Theoretically, quantum emulsion phases are challenging to analyze since they involve structures at length scales ranging from the inter-particle distance to mesoscopic scales. In contrast, the conventional tools of manybody physics are mostly geared toward two particle correlations, such as paired states, magnetism and charge density wave. Experimentally, quantum emulsion phases are not easy to probe since transport measurements can only provide indirect evidence about their existence. Realizing long-range interactions with systems of cold polar molecules $\frac{10}{15}$ can allow to explore emergent emulsion phases in a highly controllable setting. Moreover, in such systems few-body bound states, $\frac{16}{16}$ trimer liquid phases, $\frac{17}{17}$ and bound solitons ${ }^{18}$ have been predicted.

Here we demonstrate that the anisotropic and longrange character of dipolar interactions leads to new types of clustered crystal phases which appear at intermediate values of the interaction strength. Quantum mechanically, these phases melt into distinct "clustered Luttinger liquids" characterized by the decay of their densitydensity correlation functions. We calculate the phase diagram and study the quantum melting of the clustered phases when tuning the orientation of the dipoles. Our calculations indicate that the clustered phases can be explored under current experimental conditions.
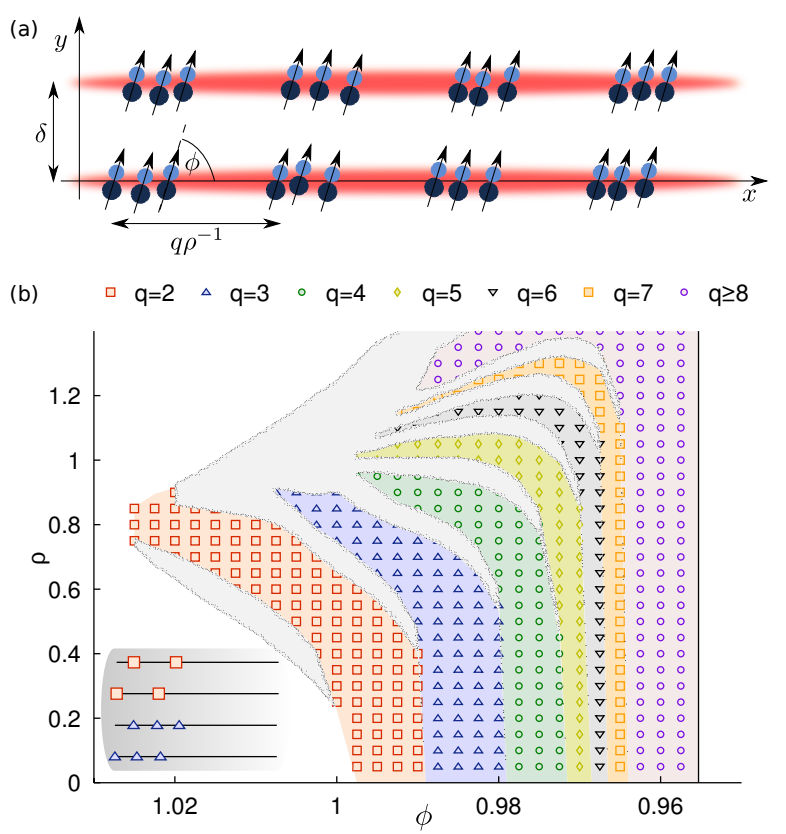

FIG. 1. (Color online) Proposed setup to observe cluster formation of polar molecules (a). The classical phase diagram for two tubes as a function of the tilting angle $\phi$ and the particle density $\rho$ is shown in (b). Lobe shaped phases consisting of clusters with $q$ particles per tube emerge. The phase separated regions are indicated by the shaded layer surrounded with speckles. Inset: Optimized cluster configuration in one unit cell for $\phi=1.01$, upper graph, and $\phi=0.99$, lower graph, at $\rho=0.7$, corresponding to $q=2$ and $q=3$, respectively.

\section{EXPERIMENTAL SETUP}

We consider a setup in which polar molecules are confined to $n_{T}$ one-dimensional parallel tubes [see Fig.11(a)], which can be realized by deep optical lattices 19 dipolar moments are aligned in the plane of the tubes at an angle $\phi$ with respect to the tube direction. The 

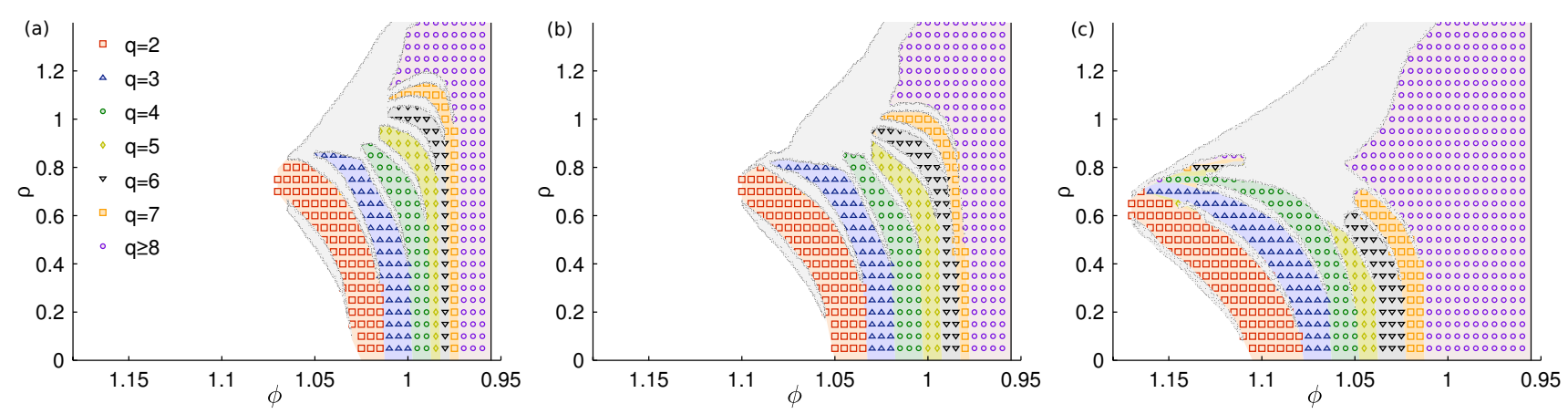

FIG. 2. (Color online) Phase diagram in the tilting angle $\phi$ and the particle density $\rho$ plane for (a) $n_{T}=3$, (b) $n_{T}=4$, and (c) $n_{T}=8$ tubes. The phase separation regions are indicated by shaded layers.

inter-tube distance $\delta$ is used as unit of length throughout this work.

The interaction energy between two molecules with dipolar moment $\mathbf{m}$ is

$$
V\left(r \mathbf{e}_{r}\right)=\frac{\mu^{2}-3\left(\mathbf{m e}_{r}\right)^{2}}{r^{3}}
$$

where $r \mathbf{e}_{r}$ is the inter-molecule displacement and $\mathbf{m}=$ $\mu(\cos \phi, \sin \phi, 0)^{T}$. For tilting angles below the critical angle $\phi_{c}=\arccos 1 / \sqrt{3}$, the interaction between molecules in the same tube is attractive and the system is unstable. Thus, we focus on dipolar orientations $\pi / 2 \geq \phi \geq \phi_{c}$ where the intra-tube interaction is repulsive. Yet, molecules in different tubes attract when their displacement along the tubes is not too large. It is precisely this interplay between attraction and repulsion which leads to the formation of clusters.

\section{CLASSICAL LIMIT}

We first discuss the emergence of mesoscopic structures in the classical limit $(\hbar \rightarrow 0)$.

When the dipoles are oriented perpendicular to the tubes $(\phi=\pi / 2)$ the ground state is a Wigner crystal with $n_{T}$ molecules per unit cell and periodicity $\rho^{-1}$, where $\rho$ is the linear density of molecules. Upon tilting the direction of the dipoles toward the critical angle $\phi_{c}$, phases with an increasingly complex unit cell are formed, before eventually becoming unstable to collapse at $\phi=\phi_{c}$. In these phases, the unit cell consist of $q$ particles per tube forming a superlattice with periodicity $q \rho^{-1}$. For example, a phase with $n_{T}=2$ and $q=3$ is illustrated schematically in Fig.11(a).

We derived the phase diagram as a function of the tilting angle $\phi$ and the density $\rho$, by minimizing the classical ground state energy with respect to the position of the molecules, allowing for arbitrary periodic structures with up to $q=8$ molecules per unit cell in each tube. The phase diagram for $n_{T}=2$ is shown in Fig.11(b).
At small densities we observe transitions to phases with monotonically increasing $q$ when decreasing the tilt angle from $\pi / 2$ toward $\phi_{c}$. Phases of a fixed value of $q$ have a lobe-like structure, which bend with increasing density toward larger $\phi$. Quite generally phases in Fig. 1. (b) terminate by phase separated regions, indicated by a shaded layer surrounded with speckles. The phase separated regions are determined by the Maxwell construction which is applicable when the interfacial energy is positive. ${ }^{4}$ It is possible that phases with $q>8$, not captured by our present calculation, are favorable in some parts of the phase diagram. In particular, this is the case very close to $\phi=\phi_{c}$ where we find that $q=8$ has the lowest energy.

The origin of the cluster formation can be easily understood by considering the case $\phi=\phi_{c}$. Then, the intratube repulsion is precisely zero. In order to maximize inter-tube attraction, it is favorable to form a single cluster with a macroscopic number of particles, corresponding to a $q \rightarrow \infty$ phase. As the angle is tuned toward $\phi_{c}$, there must be either an infinite sequence of transitions to increasingly higher values of $q$, or a macroscopically phase separated region.20

Next, we discuss systems with more than two tubes. Results for $n_{T}=3,4$, and 8 tubes are shown in Fig.2. The phase diagrams for $n_{T}>2$ have a similar lobe structure as in the $n_{T}=2$ case. The main difference is that with increasing number of tubes the lobes extend to higher values of the tilting angle $\phi$. Thus, clustered phases might be easier to observe in systems with a larger number of tubes. As in the $n_{T}=2$ case, phase separated regions appear between phases of different $q$.

A two-dimensional system which consists of an infinite number of tubes with dipoles aligned in the plane exhibits similar physics: the $q=1$ Wigner crystal phase becomes locally unstable for $\phi>\phi_{c}$. However, in this case, trial configurations with an increasing $q$ have monotonically lower energy (we have tried structures with up to $q=128$ ), indicating that the ground state may be phase separated. In the low density limit, the dipoles form infinitely long strings, which are mutually attractive ${ }^{20}$ and thus one can show that the system is unstable 
to macroscopic phase separation. Note that, for in-plane dipoles, the (logarithmically divergent) surface energy is positive, ${ }^{20}$ therefore macroscopic phase separation is possible (unlike the out-of-plane case 4).

\section{QUANTUM MECHANICAL ANALYSIS}

In quantum mechanical systems with continuous translational symmetry, true long-range crystalline order appears only in two dimensions or higher, even at zero temperature. In one-dimensional systems, the densitydensity correlations decay for large distances as a power law. Nevertheless, one can expect that upon melting the clustered Wigner crystal phases by quantum fluctuations, these phases will remain distinguishable by the nature of their quasi-long range correlations. We term the resulting phases "clustered Luttinger liquids."

In a clustered Luttinger liquid phase, the slowestdecaying component of the density-density correlations has a spatial period of $\lambda=q \rho^{-1}$. In a bosonized description, the fundamental harmonic of the density operator is therefore of the form $\cos \left[2 \pi\left(x+x_{0}\right) \rho / q\right]$, where $x_{0}$ is a uniform shift of the crystalline configuration. In terms of the "counting field" $\phi(x)^{21}$ defined relative to the crystalline configuration we obtain for the bosonized density

$$
\rho(x)=\rho-\frac{1}{\pi} \nabla \phi(x)+\rho \cos \left[\frac{2 \pi \rho x}{q}+\frac{2 \phi(x)}{q}\right]+\ldots,
$$

where the dots represent higher harmonics. The factor $q^{-1}$ present in the cosine alters the power law with which the density correlations decays:

$$
\langle\rho(x) \rho(0)\rangle=\rho^{2}-\frac{K}{2 \pi^{2}} \frac{1}{x^{2}}+\frac{\rho^{2}}{2} \cos \frac{2 \pi \rho x}{q}\left(\frac{\alpha}{x}\right)^{\frac{2 K}{q^{2}}} .
$$

Here, $K$ is the Luttinger parameter. Microscopic considerations $^{20}$ suggest that $K \propto q$. Therefore, the exponent with which the density-density correlation function decays is proportional to $1 / q$. Phases with larger $q$ thus have a slower decay of the density-density correlation function, and are increasingly "classical" in nature.

In order to make quantitative predictions about the phase diagram in the presence of quantum fluctuations, we have investigated a system of two tubes numerically by means of density matrix renormalization group (DMRG) ${ }^{22123}$ simulations. To this end, we introduce the lattice Hamiltonian

$$
\begin{aligned}
\hat{H} & =-t \sum_{\alpha, i}\left[c_{\alpha, i}^{\dagger} c_{\alpha, i+1}+c_{\alpha, i+1}^{\dagger} c_{\alpha, i}\right] \\
& +\frac{\mu^{2}}{\delta^{3}} \sum_{i, j, \alpha, \beta}^{\prime} V_{d}[(i-j) a / \delta, \alpha-\beta] \hat{n}_{\alpha, i} \hat{n}_{\beta, j}
\end{aligned}
$$

where $c_{\alpha, i}\left(c_{\alpha, i}^{\dagger}\right)$ destroys (creates) a particle at site $i$ of tube $\alpha=1,2$, and $\hat{n}_{\alpha, i}=c_{\alpha, i}^{\dagger} c_{\alpha, i}$ counts the number
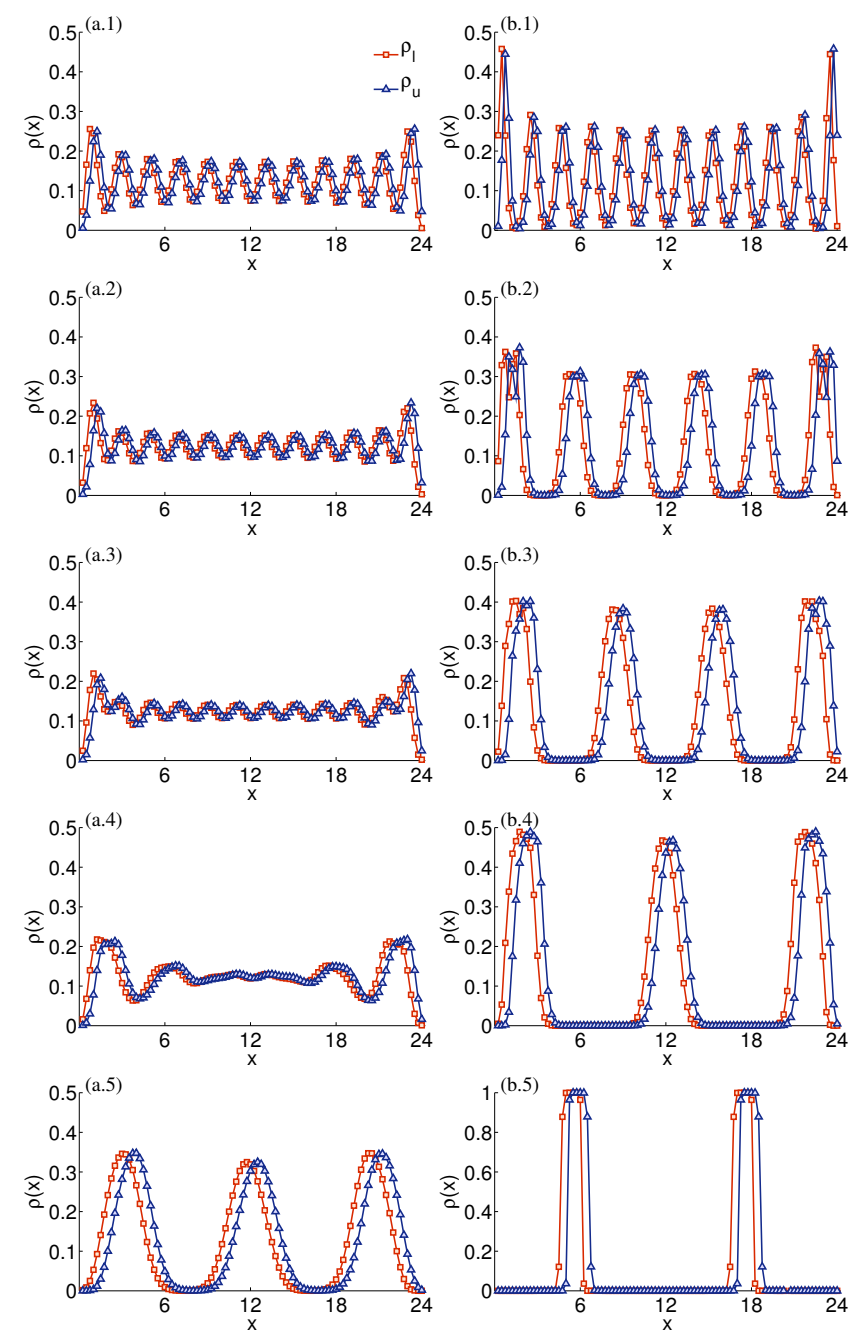

FIG. 3. (Color online) Particle density $\rho(x)$ in the lower (red squares) and the upper (blue triangles) tube for dipolar strength $\gamma=8$, left column, and $\gamma=50$, right column, for a system of length $L=24 \delta$, density $\rho=0.5 \delta^{-1}$ and lattice spacing $a=\delta / 4$. From top to bottom the tilt angle takes the values $\phi=\{1.02,0.99,0.98,0.97,0.96\}$ where for $\gamma=$ 50 pronounced clusters with $q=\{1,2,3,4,6\}$ are found, respectively. The data is obtained by DMRG for systems with open boundary conditions.

of particles. Due to the strong on-site repulsion we treat the particles as hard-core, and therefore for the quantities we compute here (e.g., density distributions and groundstate energies), it does not matter whether the particles are bosons or fermions. The discrete Hamiltonian can represent a continuous system by taking the lattice spacing $a \rightarrow 0$ while keeping the product $t a^{2}=1 /(2 m)$ constant, where $t$ is the hopping strength and $m$ is the mass of the particles in the continuum. The primed sum indicates that the singular contribution where $i=j$ and simultaneously $\alpha=\beta$ is omitted. The dipolar energy $V_{d}(x, y)$ is given by Eq. (1) with $\mathbf{r}=(x, y)^{T}$.

It is convenient to introduce the dimensionless quan- 


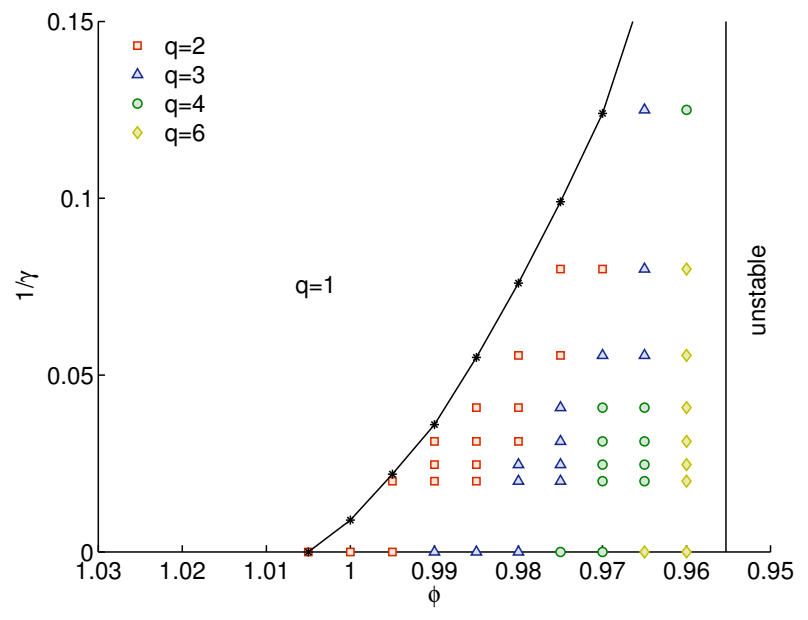

FIG. 4. (Color online) Quantum phase diagram for a two tube system of length $L=24 \delta$, density $\rho=0.5 \delta^{-1}$, and lattice spacing $a=\delta / 4$, as a function of the tilting angle $\phi$ and the ratio between the kinetic and the interaction energy $1 / \gamma$. Right of the asterisks connected by lines cluster formation can be observed. To the left of this line, the ground state is a $q=1$ Luttinger liquid.

tity $\gamma$, which is the ratio between the typical dipolar interaction energy $E_{\mathrm{d} i p}$ and the typical kinetic energy $E_{\mathrm{kin}}$. These energies can be estimated as $E_{\mathrm{d} i p} \sim \mu^{2} \rho^{3}$ and $E_{\text {kin }} \sim \rho^{2} / m$, respectively, and thus $\gamma \sim \mu^{2} \rho m$. In the limit of strong interactions, $\gamma \gg 1$, the system is expected to be essentially classical, and the phase diagram is expected to be similar to that of Fig.1 with the Wigner crystalline phases replaced by clustered Luttinger liquids. Conversely, for $\gamma \ll 1$, quantum fluctuations dominate, and we expect only the $q=1$ phase to survive.

The particle density $\rho(x)$ evaluated with DMRG for the Hamiltonian (3) exhibits clear signatures of clustered phases, see Fig. 3. We consider a system of finite length $L=24 \delta$ with open boundary conditions and particle density $\rho=0.5 \delta^{-1}$ and dipolar strength $\gamma=50$. The lattice constant is $a=\delta / 4$; no significant change in the results was observed when $a$ was decreased to $\delta / 6$. Since the reflection symmetry about a plane perpendicular to the tube is broken for any tilting angle except $\phi=\pi / 2$, the density of the upper $\rho_{u}$ and the lower $\rho_{l}$ tube are slightly shifted. Additionally, we observe that the height of the peaks in the density decreases toward the trap center, consistent with quasi-long range order. Remarkably, the rate of the decay decreases strongly with increasing $q$, as expected from the Luttinger liquid analysis, Eq. (2).

The complete quantum phase diagram for the two tube system as a function of the tilting angle $\phi$ and the ratio between kinetic and interaction energy $1 / \gamma$ is shown in Fig. 4. For $1 / \gamma=0$, the results were obtained by classical minimization of the interaction energy. The DMRG simulations are used to extend the results to $1 / \gamma>0$. The phases are determined from the density distribution by calculating the number of particles localized within one cluster. The clustered Luttinger liquid phases with $q>1$ extend to considerably large values of $1 / \gamma$, making the realization of clustered phases feasible in experiments with cold dipolar molecules.

\section{EXPERIMENTAL IMPLICATIONS}

For typical densities of $\rho=10^{4} \mathrm{~cm}^{-1}, \gamma \sim$ $\{0.7,3.3,6.8,49.4,63.3\}$ can be achieved in experiments with KRb, RbCs, NaK, NaCs, and LiCs, respectively. (See 20 for details on this calculation.)

The density regime which is most favorable for observing clustered phases is $\rho \sim 0.5 \delta^{-1}-\delta^{-1}$. For $\rho \sim 10^{4} \mathrm{~cm}^{-1}$, this corresponds to an inter-tube separation of $\delta \sim 5 \cdot 10^{-5} \mathrm{~cm}$, easily attainable using an optical potential created by a laser with wavelength $\sim 1 \mu \mathrm{m}$.

$\mathrm{NaCs}$ and LiCs are thus the most promising candidates to realize clustered phases, due to their large dipolar moments. In order to make the clustered phases more robust, one can add a shallow periodic potential along the tubes. Such a periodic potential quenches the kinetic energy, thus increasing the effective value of $\gamma$. As a consequence cluster formation arises at much weaker dipolar moment also attainable by KRb.

Other effects that can be important for experiments are (i) the incommensurability of the particle number with the cluster size, (ii) the shallow trap potential along the tube direction, (iii) the strong but finite transverse confinement, (iv) quantum fluctuations in the orientation of the dipoles, and (v) finite temperature effects. Cluster formation is extremely stable with respect to (i) and (ii)!20 Incommensurability leads to a slight rearrangement of clusters and the consequence of the shallow trap along the tubes is merely that the distance between the clusters is reduced. One-dimensional tubes are realized by strong transverse confinement potentials (iii). Therefore, we consider the interactions computed for molecules with transverse wavefunctions, corresponding to a parabolic confinement, and compare them to the bare, one-dimensional interactions. The renormalization of the interactions due to the transverse confinement can be evaluated from a multipole expansion yielding $\Delta E_{\text {dip }}^{\perp} / E_{\text {dip }} \lesssim \sigma^{2} / \delta^{2}$, where $\sigma$ is the spread of the wave function in transverse direction.20 Under standard experimental conditions $\sigma \sim 25 \mathrm{~nm}$ and thus $\Delta E_{\text {dip }}^{\perp} / E_{\text {dip }} \lesssim 0.0025$. This ratio has to be compared with the relative energy difference between the clustered $q>1$ and the uniform $q=1$ phase, which typically is $0.2-0.5 \gg \Delta E_{\text {dip }}^{\perp} / E_{\text {dip }}$. Therefore, the renormalization of the interaction energy due to the finite strength of the transverse confinement is no obstacle for the observability of the clustered phases. The quantum fluctuations of the dipoles around the orientation of the electric field (iv) renormalize the dipolar potential by $\Delta E_{\text {dip }}^{e} / E_{\text {dip }} \lesssim R_{e} / \delta \sim 0.001$, where $R_{e}$ is the bond length of the molecule. Thus, this effect is also small. The temperature scale $(\mathrm{v})$ below which we expect strong 
tendency toward cluster formation is proportional to the dipolar energy $E_{\mathrm{d} i p}$. In units of the Fermi temperature $T_{F} \sim E_{\text {kin }}$ the crossover temperature is $T_{\text {cross }}=\alpha \gamma$, where the proportionality constant $\alpha$ can be estimated from the relative energy difference of the uniform and the clustered phases, i.e., $\alpha \sim 0.2-0.5$. Depending on the molecule ${ }^{201}$ the crossover temperature is $T_{\text {cross }} \gtrsim T_{F}$.

\section{CONCLUSIONS AND OUTLOOK}

In summary, the formation of clustered phases of polar molecules is a distinct consequence of the long-range and anisotropic nature of their interactions. We find that the clustered phases can be explored under current experimental conditions. A variety of techniques can be employed to observe the clustered phases, including elastic light scattering, $\stackrel{24}{ }$ noise correlations in timeof-flight images, $\frac{25}{, 25}$ and optical quantum nondemolition detection! ${ }^{16] 26}$ Cluster formation should also contribute additional dissociation resonances in lattice modulation experiments ${ }^{27}$ and $\mathrm{rf}$ spectroscopy $[28]$

A particular exciting direction for future research is to study excitations in this system. For the simple $q=1$ phase, the unit cell consists of a single particle per tube and thus only the acoustic mode exists. However, in clustered crystal phases with $q>1$ also optical branches should exist in the excitation spectra.

\section{ACKNOWLEDGMENTS}

We are grateful to E. G. Dalla Torre for insightful discussions about the Luttinger liquid analysis. M.K. wants to thank W. von der Linden and E. Arrigoni for fruitful discussions. The authors acknowledge support from Harvard-MIT CUA, the NSF Grants No. DMR-07-05472 and No. DMR-07-57145, the DARPA OLE program, AFOSR Quantum Simulation MURI, AFOSR MURI on Ultracold Molecules, the Austrian Science Fund (FWF) under Project No. P18551-N16 (M.K.) and within the SFB ViCoM (F41) (M.G.), as well as the Austrian Marshall Plan Foundation (M.K.). Calculations have been partly performed on the iCluster of Graz University of Technology.

\section{SUPPLEMENTARY MATERIAL}

Incommensurability and longitudinal confinement.Here, we investigate in detail the effects of the particle number incommensurability with the cluster size and of the shallow confining potential along the tube direction.

To demonstrate that the cluster formation is stable against the incommensurability of the particle number with the cluster size, we evaluate the density distribution for systems with $N=10, N=12$, and $N=14$ polar molecules per tube, respectively. The remaining system
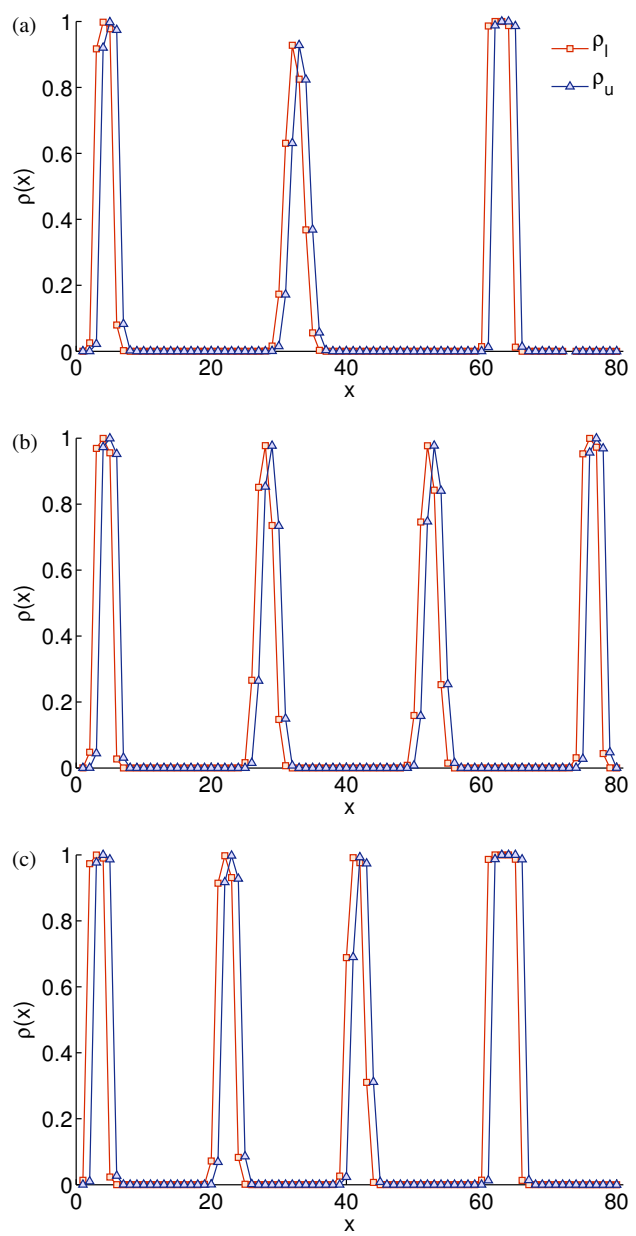

FIG. 5. (Color online) Particle density $\rho(x)$ in the lower (red squares) and in the upper (blue triangles) tube for the ratio of the interaction to kinetic energy $\gamma=2.4$, tilt angle $\phi=1$, length $L=80 \delta$, and lattice constant $a=\delta$ for systems with (a) $N=10$, (b) $N=12$, and (c) $N=14$ particles per tube. The cluster formation is insensitive as to particle densities which are incommensurable with the cluster size.

parameters are: ratio of interaction and the kinetic energy $\gamma=2.4$, tilt angle $\phi=1$, length $L=80 \delta$, and lattice constant $a=\delta$. The cluster formation is commensurable with $N=12$ particles, where four clusters with $q=3$ particles are found, see Fig.5(b). However, both $N=10$ and $N=14$ are incommensurable with the $q=3$ phase. Regardless, we observe a pronounced cluster formation, see Fig.55(a) and (c).

Next, we study the effects of the harmonic confining potential along the tube direction, which we incorporated into the Hamiltonian by a variable on-site energy $\epsilon_{j}=\mathcal{C}(j a-L / 2)^{2} / L^{2}$. It turns out that the formation of clusters is also very insensitive to the confining potential. Results for the particle distribution are shown in Fig.6 for (a) $\mathcal{C}=0.1 t$, (b) $\mathcal{C}=1.0 t$, and (c) $\mathcal{C}=4.0 t$. For the considered values of $\mathcal{C}$, the main effect of the harmonic confinement potential is that the distance between clus- 

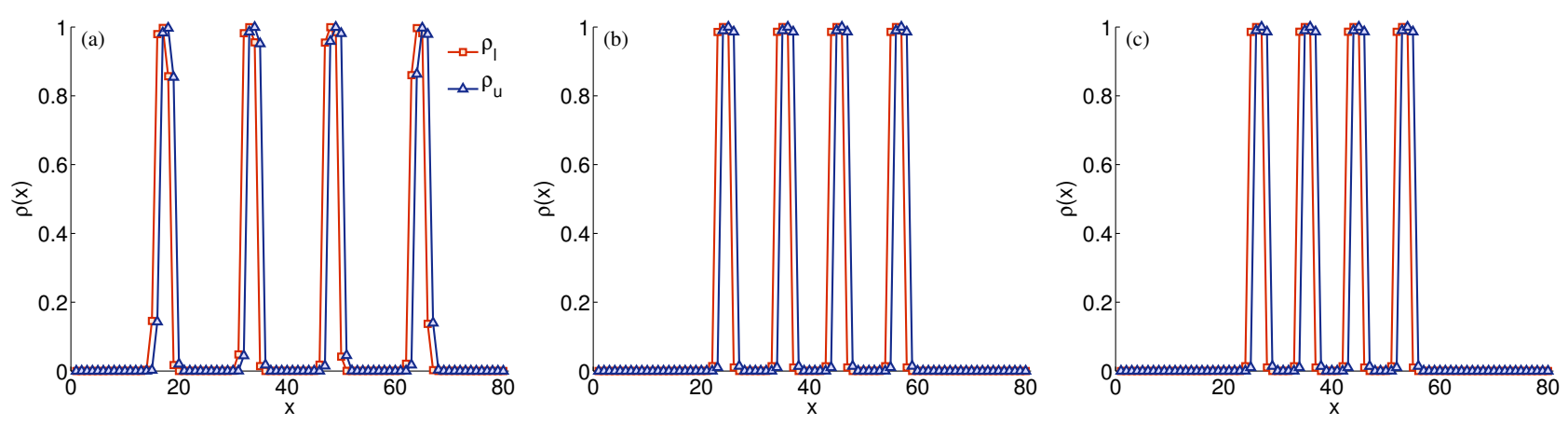

FIG. 6. (Color online) Particle density $\rho(x)$ for the same system as described in the caption of Fig. 5 but with $N=12$ throughout and a confining potential of strength (a) $\mathcal{C}=0.1 t$, (b) $\mathcal{C}=1.0 t$, and (c) $\mathcal{C}=4.0 t$. The cluster formation is robust with respect to the trap potential. The mere effect of the trap in the studied regime of the confining strength is that the distance between the clusters is reduced.

ters of particles is reduced as compared to the uniform case shown in Fig. 5 (b).

Multipole expansion. - The renormalization of the interactions due to the fluctuations of the dipoles around the orientation of the applied electric field and due to the finite spread of the transverse wavefunctions, can be evaluated from a multipole expansion of the electric potential

$$
\phi(r)=\sum_{l m} \frac{q_{l m}}{2 l+1} \frac{Y_{l m}}{r^{l+1}}
$$

where $q_{l m}=\int Y_{l m}^{*} r^{l} \rho(\mathbf{r}) d^{3} \mathbf{r}$ with the charge distribution $\rho(\mathbf{r})=\rho_{+}\left(\mathbf{r}-\mathbf{r}_{e}\right)-\rho_{-}\left(\mathbf{r}+\mathbf{r}_{e}\right)$.

The largest effect of this renormalization concerns dipoles which are separated roughly by $\delta$. The electric field induced from one dipole at the location of the second dipole is

$$
\mathbf{E}\left(\delta \mathbf{e}_{x}\right)=\nabla \phi\left(\delta \mathbf{e}_{x}\right),
$$

and the potential energy is given by $V=\mathbf{m E}\left(\delta \mathbf{e}_{x}\right)$. The leading order term is clearly the dipole-dipole interaction $E_{22}=\mathbf{m E}_{2}\left(\delta \mathbf{e}_{x}\right)$.

The next order is the dipole-quadrupole interaction $E_{42}$, induced from the fluctuations of the dipoles around the electric field. This contribution is nonvanishing, since heteronuclear polar molecules are considered. From the multipole expansion (4) we infer the microscopic quadrupolar renormalization

$$
\frac{E_{42}}{E_{22}}=\text { const. } \frac{R_{e}}{\delta}
$$

with const. $\lesssim 1$ and $R_{e}$ the bond length of the molecule.

As the transverse confinement obeys rotational symmetry, there is no quadrupole contribution from the transverse wavefunction. Therefore, the first nonvanishing contribution due to the spread of the transverse wavefunction is of the dipole-seipole (6 charges) form $E_{62}=\mathbf{m E}_{6}\left(\delta \mathbf{e}_{x}\right)$

$$
\frac{E_{62}}{E_{22}} \lesssim \frac{\sigma^{2}}{\delta^{2}}
$$

where we used $\rho_{+}(\mathbf{r})=\rho_{-}(\mathbf{r})=q /\left(\pi \sigma^{2}\right) \exp \left[-\left(y^{2}+\right.\right.$ $\left.\left.z^{2}\right) / \sigma^{2}\right] \delta(x)$ and thus $q_{l m} \sim q R_{e} \sigma^{l-1}$.

Local stability of clustered phases. - One concern could be that the uniform $q=1$ crystalline phase may be metastable, and therefore the system might not be able to find its ground state on experimental timescales. Thus, it is useful to explicitly evaluate the stability of the uniform $q=1$ phase. A system is locally unstable if its compressibility $\kappa$ is negative. The compressibility can be evaluated from $\kappa^{-1}=\partial^{2} \varepsilon / \partial\left(\rho^{-1}\right)^{2}$, where $\varepsilon$ is the energy per particle and $\rho$ the particle density. Thus it is useful to evaluate the spinodal line, which separates the regions with positive and negative compressibility, see asterisks connected by lines in Fig.77(a). On the right hand side of the spinodal line, close to the critical angle $\phi_{c}$, there is a large region where the uniform $q=1$ phase is locally unstable. In fact, it can be shown that any clustered phase (with an arbitrary $q$ ) must become locally unstable for a sufficiently large density and sufficiently close to $\phi=\phi_{c}$.

Assume that $\phi=\phi_{c}$, such that the intra-tube interaction is zero. In the high density limit, the energy per particle is proportional to the particle density times the integrated inter-tube energy. Explicitly integrating the inter-tube interaction gives $e=-\mu^{2} \rho \sin ^{2}(\phi) / \delta^{2}$. Therefore $\kappa^{-1}<0$. As we move away from the critical angle, the repulsive intra-tube interaction gives a contribution proportional to $\left(\phi-\phi_{c}\right) \rho^{3}$. Adding these two contributions and calculating the compressibility, we get that the critical density $\rho^{\star}\left(\phi^{\star}\right)$ at which the phase becomes unstable satisfies $\rho^{\star} \propto\left(\phi^{\star}-\phi_{c}\right)^{\frac{1}{2}}$.

This argument captures the main characteristics of the spinodal line at high densities. Below a certain density, the spinodal line terminates and the phase becomes locally stable, as can be seen in Fig.77(a) for $q=1$. In Fig.7(b) we show the calculated ground state energy of 
the $q=1$ phase for $\phi=0.99$, showing the region in which $\kappa<0$. The relative extend and shape of the spinodal line for systems with a larger but finite number of tubes, as shown in Fig.2, is similar to the case of two tubes.

At the critical angle $\phi=\phi_{c}$ the ground state consists of dipoles which are arbitrarily close to each other corresponding to a $q=\infty$ phase. From this and from the analysis of the compressibility follows that, there must be either an infinite sequence of phases with increasing $q$, or a region of macroscopic phase separation between a phase of finite $q$ and vacuum upon approaching $\phi=\phi_{c}$.

Infinite number of tubes. - Next, we study the phase diagram in the case of an infinite number of tubes. We find that the $q=1$ phase is locally unstable in a region $\pi / 2>\phi>\phi_{c}$. Our results are consistent with macroscopic phase separation between the $q=1$ phase and vacuum. So unlike the case with a finite number of tubes, intermediate phases with $q>1$ are not realized.

The classical ground state energy of the twodimensional system is evaluated by Ewald summation techniques ${ }^{31} \sqrt{33}$ We allow for a unit cell containing $q$ particles. The unit cell is a parallelogram of length $q \rho^{-1}$, height $\delta$, and an arbitrary angle. In addition, for $q>1$, we assume that the particles within a unit cell are equally spaced. The angle and the intra-unit cell spacing are treated as variational parameters. The same scheme was used to optimize the ground state energy in the case of a finite number of tubes, and gave excellent results compared to a full optimization with respect to the positions of all the particles in the unit cell presented in the main text [see also inset of Fig.1(b)].

Fig. 8 shows the spinodal line for the $q=1$ phase, at which the compressibility is zero. To the right of this line, the compressibility is negative, and the $q=1$ phase is locally unstable. In the unstable regime, phases with $q>$ 1 are lower in energy than the $q=1$ phase; however, the energy decreases monotonically with increasing $q$, up to the largest $q$ that we tried $(q=128)$, and is always higher than a phase separated state with macroscopic regions of $q=1$ and vacuum. In the low density limit strings of polar molecules are formed, whose mutual interaction is attractive. Therefore, at sufficiently low densities the system always phase separates.

In two-dimensional systems with dipolar interactions, the surface tension between two phases of different density diverges logarithmically ${ }^{4}$ For dipoles pointing out of the plane, the divergent term is negative, and hence macroscopic phase separation is always unstable toward forming "microemulsion" phases with emergent, mesoscopic structures. For in-plane dipoles, however, the divergent energy is positive, and therefore macroscopic phase separation is possible.

To demonstrate this, we consider a system with a linear domain wall between two phases $A$ and $B$. The dipolar contribution to the total energy is

$$
E=\frac{1}{2} \int d \mathbf{r} \int d \mathbf{r}^{\prime} \rho(\mathbf{r}) \rho\left(\mathbf{r}^{\prime}\right) V\left(\mathbf{r}-\mathbf{r}^{\prime}\right)
$$
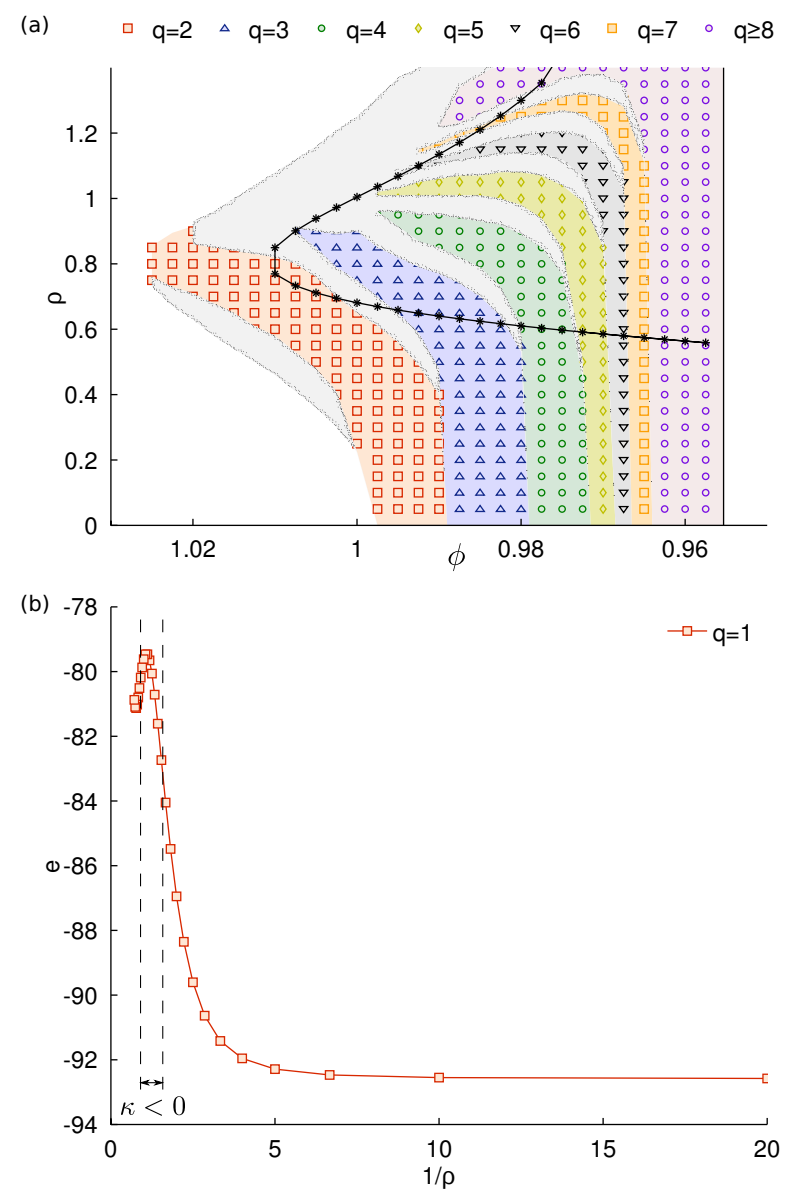

FIG. 7. (Color online) (a) Phase diagram of dipolar molecules confined in two tubes as in Fig.1 and spinodal line for the $q=1$ phase, asterisks connected by lines, which separates regions of positive and negative compressibility. (b) Ground state energy of the $q=1$ phase for $\phi=0.99$. In the region between the dashed vertical lines the curvature of the energy per particle is negative indicating the density regime where the uniform phase is certainly unstable.

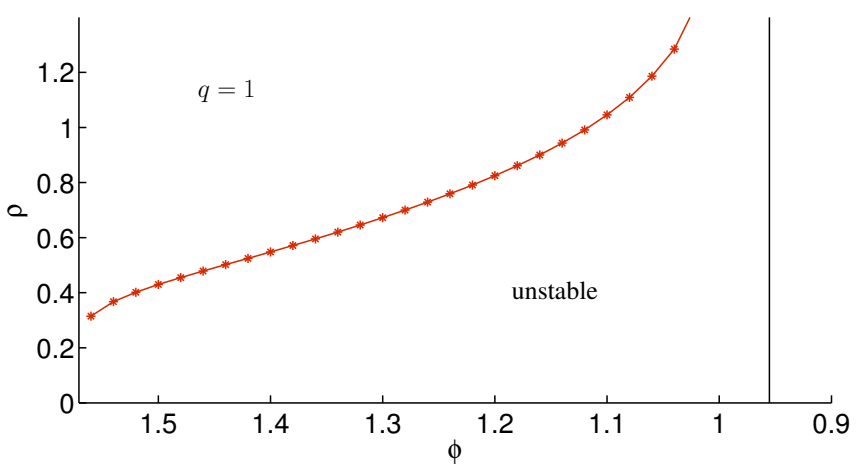

FIG. 8. (Color online) Spinodal line for a system with infinitely many tubes, which indicates the region of instability of the $q=1$ Wigner crystal phase. 
where $\rho(\mathbf{r})$ is the density at position $\mathbf{r}$, which is $\rho_{A}$ in phase $A$ and $\rho_{B}$ in phase $B$. Alternatively, the energy can be written as

$$
E=V_{A} e_{A}+V_{B} e_{B}+E_{\sigma},
$$

where $e_{A}\left(e_{B}\right)$ is the energy density of the homogeneous $A(B)$ phase, $V_{A}\left(V_{B}\right)$ is the area of phase $A(B)$, and $E_{\sigma}$ the surface energy between the two phases. The bulk contributions are defined as

$$
V_{A(B)} e_{A(B)}=\frac{1}{2} \int_{\mathbf{r} \in \Omega_{A(B)}} d \mathbf{r} \int d \mathbf{r}^{\prime} \rho_{A(B)}^{2} V\left(\mathbf{r}-\mathbf{r}^{\prime}\right),
$$

where $\Omega_{A(B)}$ defines the domain of phase $A(B)$. The integral over $\mathbf{r}^{\prime}$ is taken over the entire plane $\mathbb{R}^{2}$. Comparing Eqs. (6) and (7) yields the surface energy

$$
E_{\sigma}=-\frac{1}{2} \int_{\Omega_{A}} d \mathbf{r} \int_{\Omega_{B}} d \mathbf{r}^{\prime}\left(\rho_{A}-\rho_{B}\right)^{2} V\left(\mathbf{r}-\mathbf{r}^{\prime}\right) .
$$

For dipolar interactions, $E_{\sigma} / L$ (where $L$ is the linear dimension of the system) diverges as $\log (L)$ as $L \rightarrow \infty$. In the following, we determine the sign of $E_{\sigma}$ for a straight domain wall oriented along the $y$-direction. In order to make contact to the results of Ref. 4 where the dipoles are pointing out of plane, we consider a general dipole orientation:

$$
\mathbf{m}=\mu(\cos \phi \cos \theta, \sin \phi \cos \theta, \sin \theta)^{T},
$$

where the angle $\theta$ is measured from the plane. An explicit calculation reveals that the surface energy vanishes at an angle $\theta_{c}$ given by

$$
\theta_{c}=\arccos \sqrt{\frac{1}{1+\cos ^{2} \phi}} .
$$

For $\theta>\theta_{c}, E_{\sigma}<0$, and macroscopic phase separation is impossible; for $\theta<\theta_{c}, E_{\sigma}>0$ and phase separation is allowed. In particular, for $\theta=\pi / 2, E_{\sigma}<0$ (consistently with Ref. (4), whereas for $\theta=0, E_{\sigma} \geq 0$ for any value of $\phi$.

In summary, in the case of a two-dimensional array of tubes, it seems that the $q=1$ phase terminates at a first-order transition to vacuum, and no higher $q$ phases exist.

Numerical values for $\gamma$.-The numerical data used to evaluate the ratio between the dipolar and the kinetic energy $\gamma$ is summarized in Tab. [1.

Luttinger parameter $K$.- In order to reveal the dependence of the Luttinger parameter $K$ of the clustered system on $q$, we relate it to the Luttinger parameter $K_{0}$ of the uniform $(q=1)$ system. The low-energy properties of a $q=1$ liquid of polar molecules in one-dimensional tubes are described by $21 / 39$

$$
\begin{aligned}
H_{q=1} & =\int d x \rho_{0} \frac{(\nabla \Theta)^{2}}{2 m}+\frac{\mu^{2} \rho_{0}^{2}}{\pi^{2}}(\nabla \phi)^{2} \\
& =\frac{u_{0}}{2 \pi} \int d x\left[K_{0}(\nabla \Theta)^{2}+\frac{1}{K_{0}}(\nabla \phi)^{2}\right],
\end{aligned}
$$

TABLE I. Numerical values for the ratio between the dipolar and the kinetic energy $\gamma$ for dipoles $A B$ and the quantities which are necessary to estimate this ratio. The dipole strength is denoted as $\mu$ and $m_{A}\left(m_{B}\right)$ is the mass of constituent $A(B)$. We assume the linear density to be $\rho=$ $10^{4} \mathrm{~cm}^{-1}$.

\begin{tabular}{crrrr}
\hline \hline $\mathrm{AB}$ & $\mu / D e$ & $m_{A} / u$ & $m_{B} / u$ & $\gamma$ \\
\hline $\mathrm{KRb}^{34}$ & 0.6 & 39.1 & 85.5 & 0.7 \\
$\mathrm{RbCs}^{35}$ & 1.0 & 85.5 & 132.9 & 3.3 \\
$\mathrm{NaK}^{36}$ & 2.7 & 23.0 & 39.1 & 6.8 \\
$\mathrm{NaCs}^{37}$ & 4.6 & 23.0 & 132.9 & 49.4 \\
$\mathrm{LiCs}^{38}$ & 5.5 & 6.9 & 132.9 & 63.3 \\
\hline \hline
\end{tabular}

where we introduced the microscopic Luttinger parameters $K_{0}$ and $u_{0}$ in the second line. The potential energy contribution is given by the particle density $\rho$ times the dipolar energy per particle $\sim \mu^{2} \rho^{3}$. This gives in total the contribution $\sim \mu^{2} \rho^{4}$ to the energy density. Expanding the density using $\rho=\rho_{0}+\delta \rho$ up to second order in $\delta \rho$ gives $\rho^{4} \sim \rho_{0}^{2} \delta \rho^{2}=\rho_{0}^{2}(\nabla \phi / \pi)^{2}$. The clustered state of polar molecules has the same kinetic energy but a reduced potential energy

$$
\begin{aligned}
H_{q} & =\int d x \rho_{0} \frac{(\nabla \Theta)^{2}}{2 m}+\frac{\mu^{2} \rho_{0}^{2}}{\pi^{2} q^{2}}(\nabla \phi)^{2} \\
& =\frac{u}{2 \pi} \int d x\left[u K(\nabla \Theta)^{2}+\frac{u}{K}(\nabla \phi)^{2}\right],
\end{aligned}
$$

as the potential energy is now given by the density of clusters $\rho / q$ times the interaction energy of a single cluster $(q \mu)^{2} /(q / \rho)^{3}$. This gives rise to the additional factor $1 / q^{2}$. Comparing the Luttinger parameters in the $q=1$ and $q>1$ cases gives

$$
u_{0} K_{0}=u K \quad \text { and } \quad \frac{1}{q^{2}} \frac{u_{0}}{K_{0}}=\frac{u}{K},
$$

leading to

$$
K=q K_{0} \quad \text { and } \quad u=u_{0} / q .
$$

Therefore the Luttinger parameter $K$ of the clustered Luttinger liquid is proportional to $q$ and the decay exponent of the density-density correlation function is effectively suppressed by $1 / q$, see discussion below Eq. (2). 
${ }^{2}$ V. I. Marchenko, Zh. Eksp. Teor. Fiz. 90, 2241 (1986).

${ }^{3}$ R. E. Rosenweig, Ferrohydrodynamics (Cambridge University Press, Cambridge, 1985).

${ }^{4}$ B. Spivak and S. A. Kivelson, Phys. Rev. B 70, 155114 (2004).

${ }^{5}$ B. Spivak, S. V. Kravchenko, S. A. Kivelson, and X. P. A. Gao, Rev. Mod. Phys. 82, 1743 (2010).

${ }^{6}$ A. A. Koulakov, M. M. Fogler, and B. I. Shklovskii, Phys. Rev. Lett. 76, 499 (1996).

7 R. Moessner and J. T. Chalker, Phys. Rev. B 54, 5006 (1996).

8 M. M. Fogler, in High Magnetic Fields: Applications in Condensed Matter Physics and Spectroscopy (Springer, Berlin, 2002) 1st ed., pp. 98-138.

9 J. P. Eisenstein, K. B. Cooper, L. N. Pfeiffer, and K. W. West, Phys. Rev. Lett. 88, 076801 (2002).

${ }^{10}$ L. Santos, G. V. Shlyapnikov, P. Zoller, and M. Lewenstein, Phys. Rev. Lett. 85, 1791 (2000).

11 M. A. Baranov, M. S. Marenko, V. S. Rychkov, and G. V. Shlyapnikov, Phys. Rev. A 66, 013606 (2002).

${ }^{12}$ J. Doyle, B. Friedrich, R. V. Krems, and F. MasnouSeeuws, Eur. Phys. J. D 31, 149 (2004).

13 M. Baranov, Phys. Rep. 464, 71 (2008).

${ }^{14}$ L. D. Carr, D. DeMille, R. V. Krems, and J. Ye, New J. Phys. 11, 055049 (2009).

15 T. Lahaye, C. Menotti, L. Santos, M. Lewenstein, and T. Pfau, Rep. Prog. Phys. 72, 126401 (2009).

${ }^{16}$ B. Wunsch, N. T. Zinner, I. B. Mekhov, S. J. Huang, D. W. Wang, and E. Demler, Phys. Rev. Lett. 107, 073201 (2011).

17 M. Dalmonte, P. Zoller, and G. Pupillo, Phys. Rev. Lett. 107, 163202 (2011).

${ }^{18}$ M. Bauer and M. M. Parish, Phys. Rev. Lett. 108, 255302 (2012).

19 A. Chotia, B. Neyenhuis, S. A. Moses, B. Yan, J. P. Covey, M. Foss-Feig, A. M. Rey, D. S. Jin, and J. Ye, Phys. Rev. Lett. 108, 080405 (2012).

${ }^{20}$ See supplementary material.

21 T. Giamarchi, Quantum Physics in One Dimension (Oxford University Press, USA, 2004).
${ }^{22}$ S. R. White, Phys. Rev. Lett. 69, 2863 (1992).

${ }^{23}$ U. Schollwöck, Rev. Mod. Phys. 77, 259 (2005).

${ }^{24}$ C. A. Müller, T. Jonckheere, C. Miniatura, and D. Delande, Phys. Rev. A 64, 053804 (2001).

${ }^{25}$ E. Altman, E. Demler, and M. D. Lukin, Phys. Rev. A 70, 013603 (2004).

${ }^{26}$ N. T. Zinner, B. Wunsch, I. B. Mekhov, S. J. Huang, D. W. Wang, and E. Demler, Phys. Rev. A 84, 063606 (2011).

27 T. Stöferle, H. Moritz, C. Schori, M. Köhl, and T. Esslinger, Phys. Rev. Lett. 92, 130403 (2004).

${ }^{28}$ C. A. Regal and D. S. Jin, Phys. Rev. Lett. 90, 230404 (2003).

29 S. Gupta, Z. Hadzibabic, M. W. Zwierlein, C. A. Stan, K. Dieckmann, C. H. Schunck, E. G. M. van Kempen, B. J. Verhaar, and W. Ketterle, Science 300, 1723 (2003).

30 C. A. Regal, C. Ticknor, J. L. Bohn, and D. S. Jin, Nature (London) 424, 47 (2003).

31 P. P. Ewald, Ann. Phys. 369, 253 (1921).

${ }^{32}$ U. Essmann, L. Perera, M. L. Berkowitz, T. Darden, H. Lee, and L. G. Pedersen, J. Chem. Phys. 103, 8577 (1995).

33 A. Grzybowski, E. Gwóźdź, and A. Bródka, Phys. Rev. B 61, 6706 (2000).

${ }^{34} \mathrm{~K} . \mathrm{Ni}$, S. Ospelkaus, M. H. G. de Miranda, A. Pe'er, B. Neyenhuis, J. J. Zirbel, S. Kotochigova, P. S. Julienne, D. S. Jin, and J. Ye, Science 322, 231 (2008).

${ }^{35}$ M. Debatin, T. Takekoshi, R. Rameshan, L. Reichsöllner, F. Ferlaino, R. Grimm, R. Vexiau, N. Bouloufa, O. Dulieu, and H. Nägerl, Phys. Chem. Chem. Phys. 13, 18926 (2011).

${ }^{36}$ J. W. Park, C. Wu, I. Santiago, T. G. Tiecke, P. Ahmadi, and M. W. Zwierlein, Phys. Rev. A 85, 051602(R) (2012).

37 C. Haimberger, J. Kleinert, O. Dulieu, and N. P. Bigelow, J. Phys. B: At. Mol. Opt. Phys. 39, S957 (2006).

38 J. Deiglmayr, M. Repp, A. Grochola, K. Mörtlbauer, C. Glück, O. Dulieu, J. Lange, R. Wester, and M. Weidemüller, Faraday Discuss. 142, 335 (2009).

${ }^{39}$ C. Kollath, J. S. Meyer, and T. Giamarchi, Phys. Rev. Lett. 100, 130403 (2008). 